\title{
TIM-3 expression in lymphoma cells predicts chemoresistance in patients with adult T-cell leukemia/lymphoma
}

\author{
HASITA HORLAD $^{1 *}$, KOJI OHNISHI ${ }^{*}$, CHAOYA MA $^{1}$, YUKIO FUJIWARA ${ }^{1}$, DAISUKE NIINO ${ }^{2}$, \\ KOICHI OHSHIMA ${ }^{2}$, MASAHISA JINUSHI ${ }^{3}$, MASAO MATSUOKA $^{4}$, \\ MOTOHIRO TAKEYA ${ }^{1}$ and YOSHIHIRO KOMOHARA ${ }^{1}$ \\ ${ }^{1}$ Department of Cell Pathology, Graduate School of Medical Sciences, Kumamoto University, \\ Kumamoto 860-8556; ${ }^{2}$ Department of Pathology, School of Medicine, Kurume University, \\ Kurume, Fukuoka 839-0851; ${ }^{3}$ Institute for Advanced Medical Research, Graduate School of Medicine, \\ Keio University, Tokyo 108-8345; ${ }^{4}$ Institute for Virus Research, Kyoto University, \\ Kyoto 606-8501, Japan
}

Received April 4, 2015; Accepted May 23, 2016

DOI: $10.3892 / \mathrm{ol} .2016 .4774$

\begin{abstract}
Adult T-cell leukemia/lymphoma (ATLL), an aggressive type of malignant lymphoma, is highly resistant to chemotherapy. However, the detailed mechanisms of the chemoresistance of ATLL have never been elucidated. We previously demonstrated that direct cell-cell interaction between macrophages and lymphoma cells was significantly associated with lymphoma progression in patients with ATLL. The present study aimed to further analyze the effects of cell-cell interaction between macrophages and ATLL cells by means of cell culture studies and immunohistochemical analysis using human ATLL samples. It was found that direct co-culture with macrophages induced chemoresistance in the ATLL ATN-1 cell line, but not in other cell lines, including TL-Mor, ED and ATL-2S. It was also found that expression of the $\mathrm{T}$ cell $\mathrm{Ig}$ and mucin domain-containing molecule- 3 (TIM-3) was induced in ATN-1 cells by their long-term co-culture with macrophages. TIM-3 gene transfection induced chemoresistance in the ATN-1 cells. Immunostaining of ATLL tissues showed TIM-3 expression in 25 out of 58 ATLL cases. Although TIM-3 expression was not associated with overall survival or $\mathrm{T}$ classification, it was associated with resistance to chemotherapy. TIM-3 expression is therefore considered to be a marker for predicting the
\end{abstract}

Correspondence to: Dr Yoshihiro Komohara, Department of Cell Pathology, Graduate School of Medical Sciences, Kumamoto University, 1-1-1 Honjo, Chūō, Kumamoto 860-8556, Japan

E-mail: ycomo@kumamoto-u.ac.jp

*Contributed equally

Key words: TIM-3, macrophage, adult T-cell leukemia/lymphoma, chemoresistance, cluster of differentiation 163 efficacy of chemotherapy, and TIM-3-associated signals may be a therapeutic target for patients with ATLL.

\section{Introduction}

Adult T-cell leukemia/lymphoma (ATLL) is one histological type of aggressive lymphoma that is endemic in Japan (southwestern region), the Caribbean basin and Central Africa (1). ATLL is caused by human T-cell leukemia virus type I (HTLV-1) infection, and the HTLV-1 p40 tax protein and HTLV-1 basic leucine zipper factor are considered to be important for oncogenesis (2). Since ATLL cells are generally resistant to chemotherapy, the median survival time of acute and lymphomatous ATLL is reported to be $\sim 1$ year (3). It is therefore a matter of great urgency to understand the resistance of ATLL cells to chemotherapy at a molecular level in order to uncover useful targets for the therapy of ATLL.

$\mathrm{T}$ cell Ig and mucin domain-containing molecule-3 (TIM-3), also known as hepatitis A virus cellular receptor 2 , is widely expressed on immune cells such as monocyte/ macrophages, dendritic cells, natural killer cells and $\mathrm{T}$ cells $(4,5)$. Galectin-9 and high mobility group box 1 are known to be the ligands of TIM-3, and TIM-3 signaling is generally involved in the regulation of immune responses by negatively regulating interferon secretion (6). Recently, TIM-3 has also been found to be expressed in melanoma, liver cancer and lung cancer, and higher expression of TIM-3 has been shown to be associated with a poor clinical prognosis in these cancers (7-10).

We previously demonstrated that macrophages induced ATLL cell proliferation and that a higher density of tumor-associated macrophages (TAMs) in ATLL tissues was closely associated with a poor clinical prognosis (11). It was also found that direct contact with macrophages induced ATLL cell activation, although the detailed mechanisms have not yet been elucidated (11).

In the present study, direct co-culture with macrophages induced chemoresistance and TIM-3 overexpression in ATLL 
cells, and the correlation between TIM-3 expression and chemoresistance was investigated.

\section{Materials and methods}

Cell lines and cell culture. The human ATLL cell lines (ATN-1, TL-Mor, ED and ATL-2s) (12) and a T-cell lymphoma cell line without HTLV-1 infection (MOLT-4) were maintained in RPMI (Wako Pure Chemical Industries, Ltd., Tokyo, Japan) supplemented with $10 \%$ fetal bovine serum. The ATN-1, TL-Mor and MOLT-4 cells were purchased from the RIKEN Cell Bank (Wako, Japan). The ED and ATL-2 cell lines were previously established by Professor Masao Matsuoka (Institute for Virus Research, Kyoto University, Kyoto, Japan). The mycoplasma test was performed by using a polymerase chain reaction $(\mathrm{PCR})$ detection kit purchased from Takara Bio Inc. (Otsu, Japan). Adriamycin (ADR) and carboplatin (CBDCA) were obtained from Wako Pure Chemical Industries, Ltd. The number of ATLL cells following 2-day culture with ADR (50 or $100 \mu \mathrm{M})$ and CBDCA $(0.3$ or $1.0 \mu \mathrm{M})$ was tested using the WST assay kit (Dojindo Molecular Technologies Inc., Kumamoto, Japan).

Macrophage culture. Peripheral blood mononuclear cells were obtained from healthy volunteer donors, and all donors supplied written informed consent for experimental use of these cells. Cluster of differentiation (CD)14-positive monocytes were isolated using CD14-microbeads (Miltenyi Biotec, Bergisch Gladbach, Germany). These monocytes were plated in 24-well plates $\left(5 \times 10^{4} /\right.$ well) and cultured with granulocyte-macrophage colony-stimulating factor (GM-CSF; $5 \mathrm{ng} / \mathrm{ml}$ ) and macrophage CSF (M-CSF; $100 \mathrm{ng} / \mathrm{ml}$ ) (Wako) for 7 days at $37^{\circ} \mathrm{C}$.

Flow cytometry (fluorescence-activated cell sorting). Phycoerythrin-labeled anti-TIM-3 antibody (mouse monoclonal; clone F382E2; \#345006; dilution, 1:50), fluorescein isothiocyanate-labeled anti-CD14 antibody (mouse monoclonal; clone HCD14; \#325604; dilution, 1:50) and isotype matched control antibodies (mouse IgG1 kappa; clone MOPC-21; \#400112 and \#400110) were purchased from BioLegend Inc. (San Diego, CA). The surface expression of these molecules on stained cell samples was analyzed using a FACSverse flow cytometer (BD Biosciences, Franklin Lakes, NJ, USA) with FACSuite (BD Biosciences) software.

TIM-3-expressing ATLL cell lines. A plasmid encoding the TIM-3 gene (pDsRed-TIM-3) and a control pDsRed plasmid (Takara Bio Inc., Otsu, Japan) were transfected into ATN-1 and ED cells using HilyMax (Dojindo Molecular Technologies Inc.). Following selection with neomycin, single cell cloning was performed. Cell clones were selected and cell surface TIM-3 expression was tested using flow cytometry.

Tissue samples. Paraffin-embedded tumor samples (thickness, $3 \mu \mathrm{m}$ ) from the lymph nodes of 58 patients with ATLL (lymphomatous and acute type) were examined. Patients diagnosed between January 1993 and December 2003 were treated with conventional chemotherapy and no patient was treated with bone marrow transplantation. A total of 29 patients showed leukemic change. Written informed consent was
Table I. TIM-3 expression and clinicopathological factors.

TIM-3, n

Factor

n Negative Positive P-value

\begin{tabular}{lrrrr}
\hline $\begin{array}{l}\text { Age, years } \\
\geq 65\end{array}$ & 29 & 17 & 12 & 0.79 \\
$<65$ & 29 & 16 & 13 & \\
Gender & & & & 0.15 \\
$\quad$ Male & 34 & 22 & 12 & \\
$\quad \begin{array}{l}\text { Female } \\
\text { Stage }\end{array}$ & 24 & 11 & 13 & \\
$\quad$ I, II, III & & & & 0.66 \\
IV & 24 & 15 & 9 & \\
$\begin{array}{l}\text { Response to chemotherapy } \\
\text { Partial/complete }\end{array}$ & 30 & 17 & 13 & \\
$\quad$ No response & 38 & 24 & 14 & \\
$\quad$ Leukemic change & 10 & 2 & 8 & \\
$\quad$ Yes & & & & 0.46 \\
$\quad$ No & 29 & 15 & 14 & \\
\hline
\end{tabular}

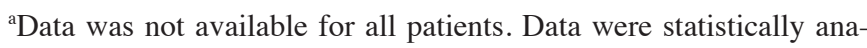
lyzed by the $\chi^{2}$ test. TIM-3, T cell Ig and mucin domain-containing molecule-3; N.S., not significant.

obtained from all patients in accordance with protocols approved by the Fukuoka University and Kurume University Review Board (Kurume, Japan). The experimental procedure was approved by the Kumamoto University Review Board (Kumamoto, Japan). We previously published characteristics of this same set of ATLL specimens, and thus, the numbers of CD68- and CD163-positive cells published in that previous report (11) were used in this study.

Immunohistochemistry. Rabbit polyclonal anti-Iba-1 (macrophage marker; \#019-19741; Wako Pure Chemical Industries, Ltd.) and goat polyclonal anti-TIM-3 (\#LS-C55594; LifeSpan Biosciences Inc., Seattle, WA, USA; dilution, 1:2,000) were used as primary antibodies. Normal goat immunoglobulin (Santa Cruz, Dallas, Texas) was used as a negative control and no signal was observed in negative control-stained sections. Each immunostaining was performed at the same time, and the reactions were visualized using the diaminobenzidine substrate system (Nichirei Bioscience, Tokyo, Japan). The data were evaluated by two investigators who were blinded to any information regarding the samples. For double-immunostaining, TIM-3 was visualized using the diaminobenzidine substrate system in the first step. After washes in glycine buffer ( $\mathrm{pH} 2.2)$, sections were then stained with the anti-Iba-1 antibody and visualized using HistoGreen solution (Linaris Biologische Produkte, Wertheim-Bettingen, Germany).

Statistical analysis. Statistical analysis of in vitro and in vivo data was performed using JMP 10 software (SAS Institute, Chicago, IL, USA). All values from in vitro studies represent 

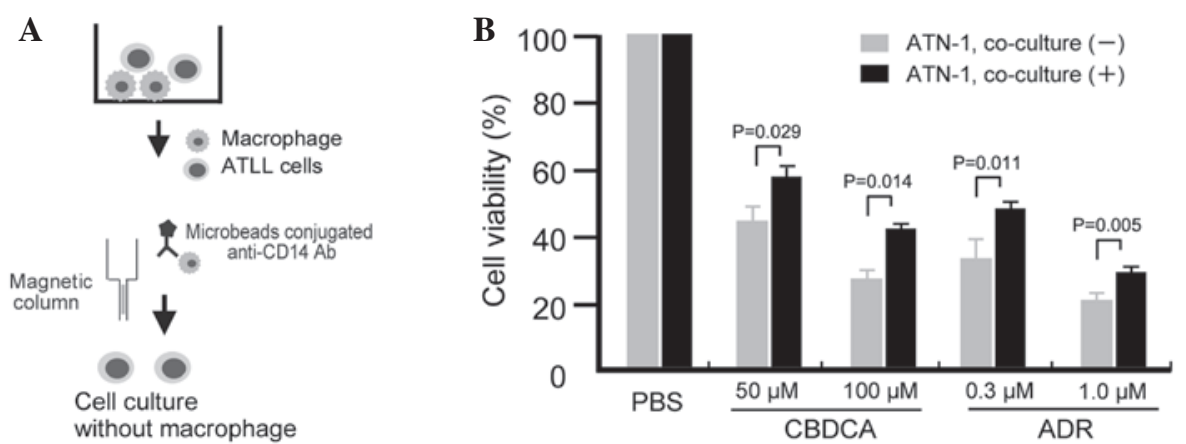

Figure 1. Chemoresistance of ATN-1 cells co-cultured with macrophages. (A) Method of adult T-cell leukemia/lymphoma (ATLL) cell isolation following co-culture with macrophages. (B) Following co-culture with macrophages for 3 weeks, the ATN-1 cells were incubated with the anticancer agents carboplatin (CBDCA) and Adriamycin (ADR) for $24 \mathrm{~h}$. The sensitivity of the ATN-1 cells to CBDCA and ADR was then evaluated using a WST assay. PBS, phosphate-buffered saline; $\mathrm{CD}$, cluster of differentiation; $\mathrm{Ab}$, antibody.

A

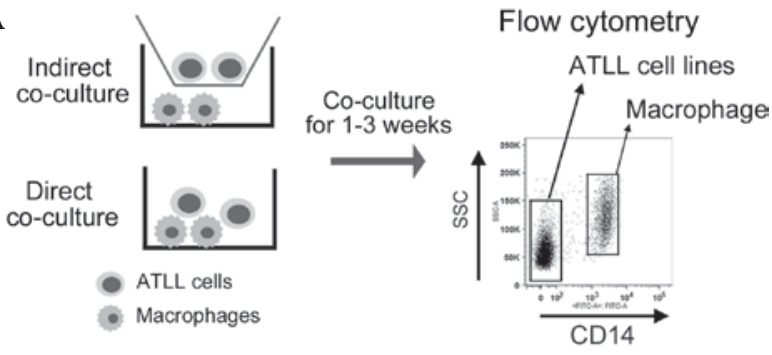

B
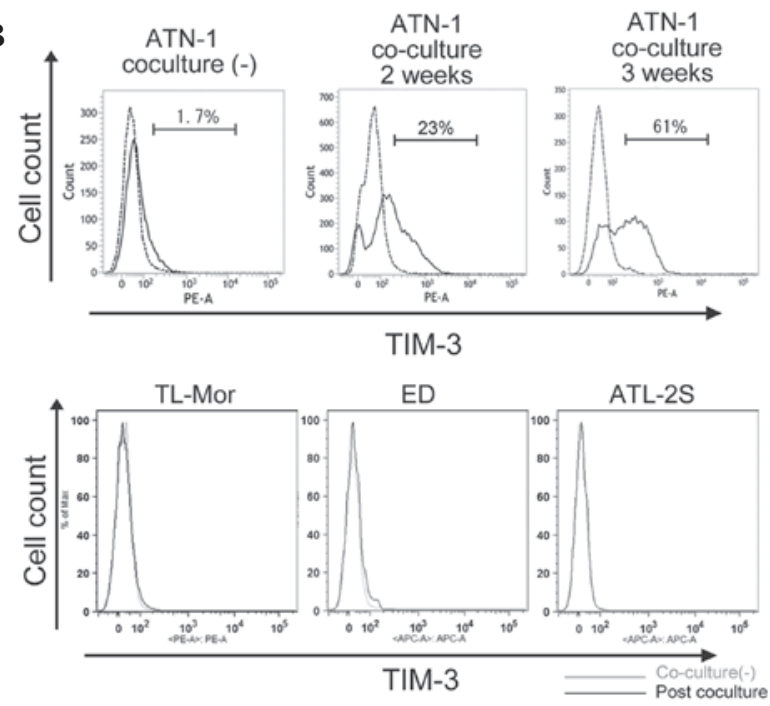

C

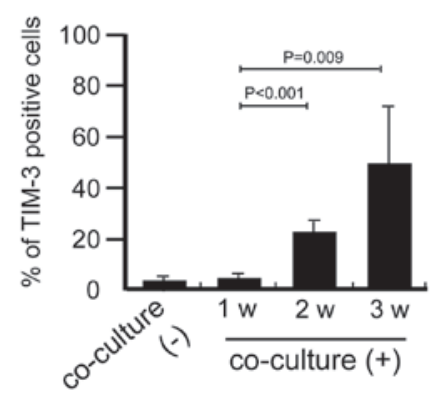

Figure 2. T cell Ig and mucin domain-containing molecule-3 (TIM-3) expression in adult T-cell leukemia/lymphoma (ATLL) cell lines. (A) Outline of the ATLL/macrophage co-culture methods used and flow cytometric analysis of the cells. (B) TIM-3 expression in the CD14-negative ATLL cell lines (ATN-1, TL-Mor, ED and ATL-2S) was evaluated pre- and post-co-culture with macrophages, using flow cytometry. (C) The percentage of TIM-3-positive ATN-1 cells was evaluated after the indicated weeks $(w)$ of co-culture with macrophages. SSC, sidewards scatter; CD, cluster of differentiation. results of 2 or 3 independent experiments. Data are expressed as the mean \pm standard deviation. Student's t-test was used for comparisons of two groups in in vitro studies. $\mathrm{P}<0.05$ was considered to indicate a statistically significant difference.

\section{Results}

Long-term co-culture with macrophages induces chemoresistance in ATN-1 cells. The study first tested whether the sensitivity of ATLL cell lines to anticancer compounds may change following their co-culture with macrophages through use of an in vitro co-culture assay. In this direct co-culture system, cells from the ATLL ATN-1, TL-Mor, ED, ATL-2s or MOLT- 4 cell lines were co-cultured with macrophages for 1,2 or 3 weeks, following which the co-cultured cells were depleted of macrophages by using microbeads conjugated to an anti-CD14 antibody and a magnetic column (Fig. 1A). Contamination of the lymphoma cells with macrophages was $<2 \%$ following this depletion procedure (data not shown). The sensitivity of the co-cultured ATLL cells to the anticancer drugs ADR or CBDCA was then assayed by evaluation of cell viability using a WST assay. The sensitivity of ATN-1 cells to ADR and CBDCA was significantly decreased by prior co-culture with macrophages for 3 weeks (all $\mathrm{P}<0.05$; Fig. 1B). Resistance of ATN-1 cells to ADR and CBDCA was also induced by 2 weeks of prior co-culture with macrophages; however, the differences in anticancer drug sensitivities between cells cultured with or without macrophages were smaller than those of the 3-week co-cultured cells (data not shown). Indirect co-culture using Transwells did not impact the sensitivity of the ATN-1 cells to ADR or CBDCA (data not shown), suggesting that direct contact between the macrophages and ATN-1 cells was required for the observed effect.

TIM-3 expression on ATN-1 cells is induced by long-term direct co-culture with macrophages. Based on preliminary cDNA microarray data (data not shown), we suspected that TIM-3 expression in ATN-1 cells was upregulated by co-culture with macrophages. To confirm this possibility, the effect of co-culture with macrophages on TIM-3 expression by ATN-1 cells was analyzed using flow cytometry. ATN-1 cells and macrophages were distinguished from each other using the anti-CD14 antibody as a macrophage marker (Fig. 2A). 

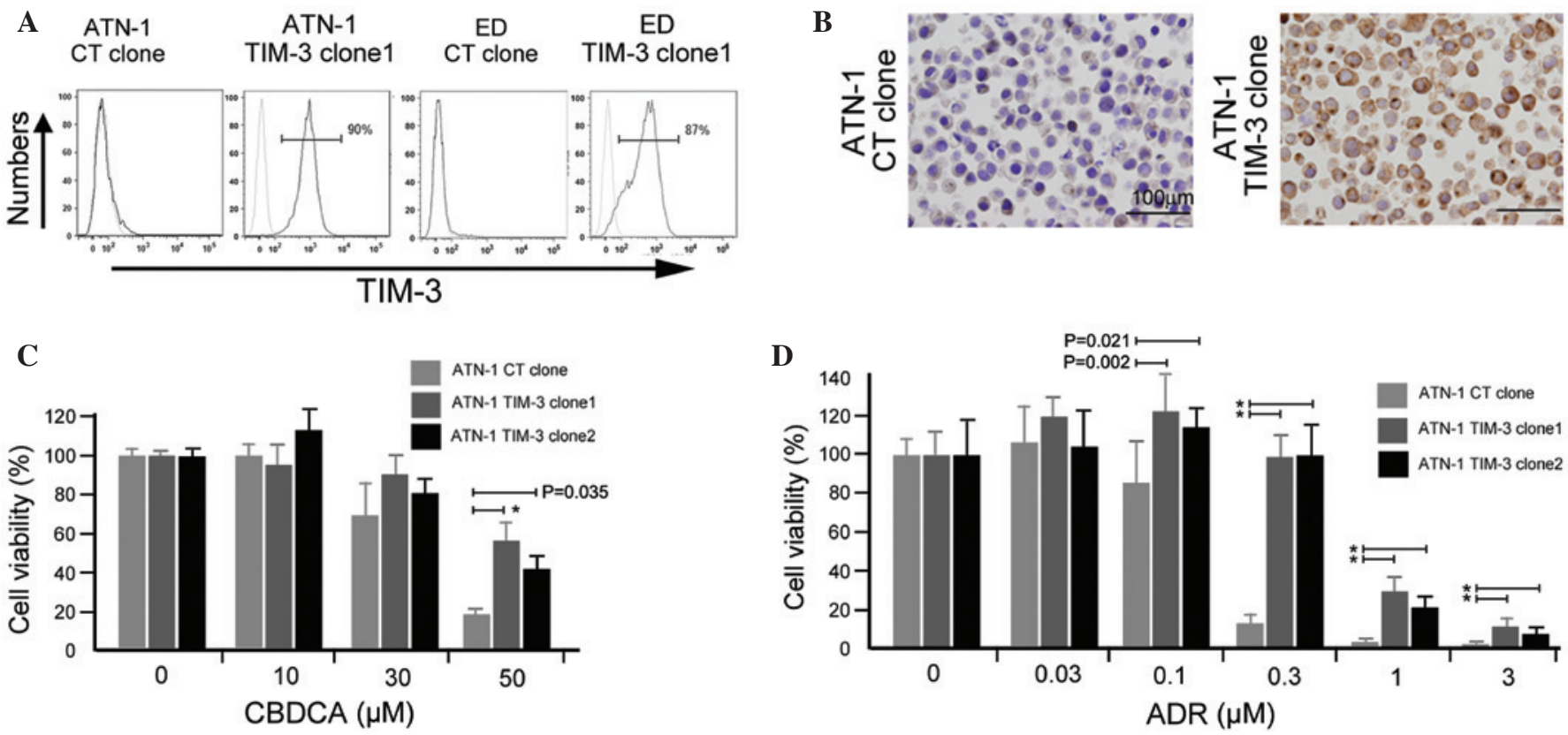

Figure 3. Involvement of T cell Ig and mucin domain-containing molecule-3 (TIM-3) in macrophage-induced chemoresistance of adult T-cell leukemia/lymphoma (ATLL) cells. ATN-1 and ED cells were transfected with a vector encoding the TIM-3 gene or a control (CT; DsRed) gene and TIM-3-expressing clones were established. TIM-3 expression was analyzed using (A) flow cytometry and (B) immunostaining. TIM-3-negative and -positive ATN-1 cloned cells (TIM-3-negative CT clone, TIM-3-positive clones 1 and 2) were cultured with the anticancer agents (C) carboplatin (CBDCA) or (D) Adriamycin (ADM) for $24 \mathrm{~h}$, and cell viability was tested using the WST assay. ${ }^{*} \mathrm{P}<0.001$.

This flow cytometric analysis showed that, although little TIM-3 expression was detected in the control ATN-1 cells or in the ATN-1 cells co-cultured with macrophages for 1 week, TIM-3 expression was significantly induced in the ATN-1 cells by 2 and 3 weeks of co-culture with macrophages $(\mathrm{P}<0.05$; Fig. 2B and C). By contrast, TIM-3 expression was not detected in, and was not induced by co-culture with macrophages in other cell lines (Fig. 2B). The induction of TIM-3 overexpression in ATLL cell lines by co-culture with macrophages was not observed in the indirect co-culture system (data not shown).

TIM-3 overexpression is involved in the chemoresistance of ATN-1 cells. Next, an in vitro experiment was performed using ATLL cell lines to confirm the involvement of TIM-3 in their chemoresistance. A TIM-3 gene construct was transfected into ATN-1 and ED cells, and TIM-3-expressing ATN-1 and ED cells were established (Fig. 3A). TIM-3 expression in established ATN-1 and ED clones was confirmed using flow cytometry (Fig. 3A) and its expression in ATN-1 clones was further confirmed by immunostaining (Fig. 3B). Two TIM-3-positive ATN-1 clones showed increased chemoresistance to ADR and CBDCA compared with control ATN-1 cells $(\mathrm{P}<0.05$; Fig. $3 \mathrm{C}$ and $\mathrm{D})$, whereas the growth rate of TIM-3-positive ATN-1 cells did not differ from that of control ATN-1 cells (data not shown). Notably, TIM-3 overexpression did not affect either the sensitivity of the ED cells to ADR and CBDCA or the growth rate of the ED cells (data not shown).

TIM-3 expression in lymphoma cells is correlated with chemoresistance in patients with ATLL. Finally, the potential clinical significance of TIM-3 expression was investigated by immunostaining for TIM-3 expression in 58 ATLL tissue specimens. Cases in which $>50 \%$ of the lymphoma cells were positive for TIM-3 were classified as TIM-3-positive. This analysis indicated that 25 out of the total 58 cases were TIM-3-positive (Fig. 4A). Since certain macrophages also stained for TIM-3, TIM-3 expression on lymphoma cells was confirmed by means of double-immunostaining. Thus, TIM-3 expression was detected in lymphoma cells that were negative for the macrophage marker Iba-1 (Fig. 4B). The association between TIM-3 expression and clinicopathological factors was then analyzed, and TIM-3-positive cases were found to be resistant to chemotherapy ( $\mathrm{P}=0.015$; Table I). However, TIM-3 expression was not associated with overall survival or other clinical factors (all $\mathrm{P}>0.05$; Fig. $4 \mathrm{C}$ and Table I).

The correlation between macrophage infiltration and TIM-3 expression in the lymphoma cells was also evaluated and a higher density of CD163-positive macrophages was detected in the TIM-3-positive cases ( $\mathrm{P}=0.020$; Fig. 4D). The density of CD68-positive macrophages appeared to be higher in the TIM-3-positive cases, however, no significant association was found ( $\mathrm{P}=0.055$; Fig. 4D).

Since the mRNA expression of TIM-3 in human ATLL samples was not evaluated in the present study, data analysis using the PubMed central database was performed to determine TIM-3 mRNA expression in ATLL. In this analysis, TIM-3 mRNA expression was observed in CD4-positive lymphocytes of patients with acute ATLL; however, no significant differences in TIM-3 mRNA expression were observed between the CD4-positive lymphocytes of healthy donors and those of patients with acute ATLL (Fig. 5A). Since no microarray dataset analysis of lymph node biopsies could be found in the database, TIM-3 expression was analyzed in a dataset of peripheral T-cell lymphoma (PTCL) samples and TIM-3 was found to be upregulated in the PTCL samples compared with the control reactive lymphadenitis samples ( $\mathrm{P}=0.01$; Fig. $5 \mathrm{~B})$. 


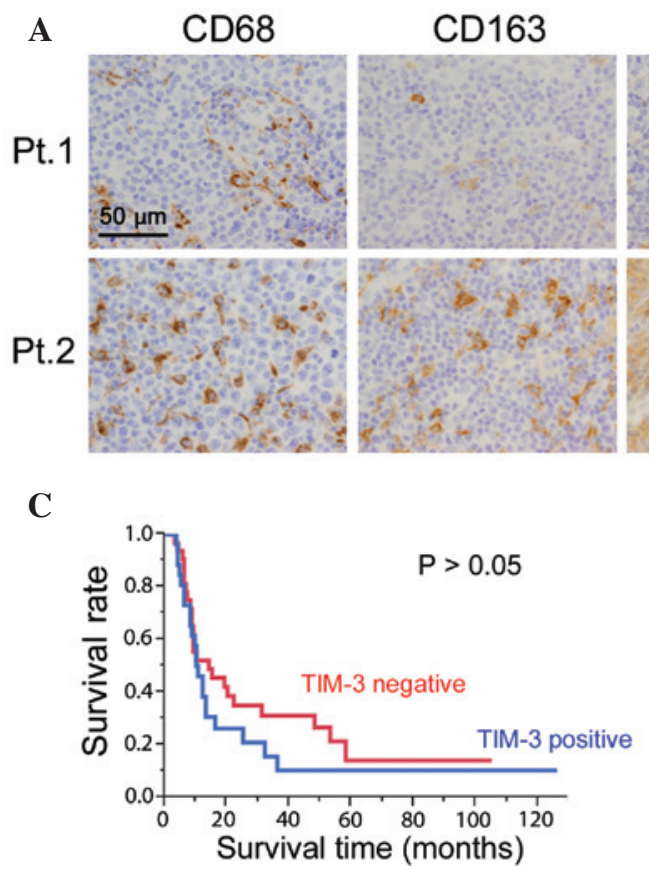

TIM-3

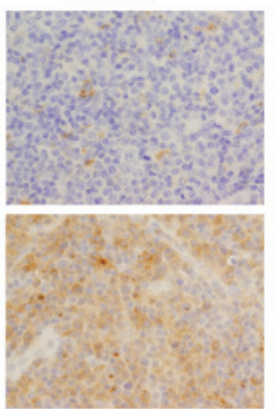

B

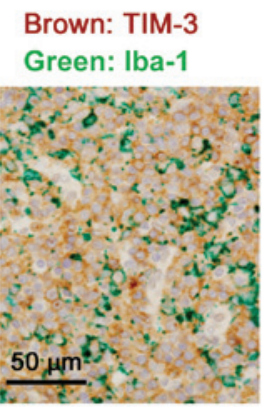

D

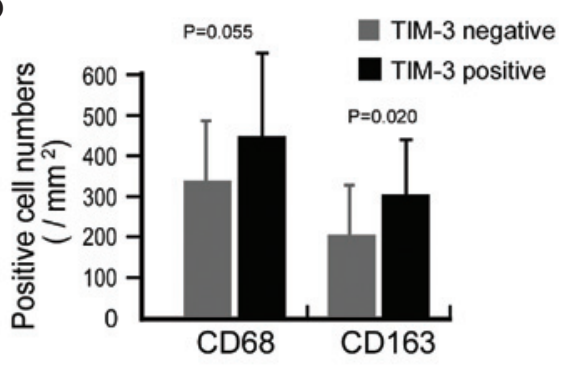

Figure 4. Immunohistochemical analysis of the expression of T cell Ig and mucin domain-containing molecule-3 (TIM-3) and macrophage markers in adult T-cell leukemia/lymphoma (ATLL) tissues. (A) Representative immunostaining of the macrophage/monocyte markers cluster of differentiation (CD)68 and CD163, and of TIM-3, in ATLL tissue of a TIM-3-negative (Pt.1) and a TIM-3-positive (Pt.2) patient. (B) Representative double-immunostaining of TIM-3 and Iba-1 (a pan-macrophage marker). (C) Survival rate of TIM-3-negative and -positive cases analyzed by Kaplan-Meier analysis. (D) The association between TIM-3 expression and tumor macrophage infiltration.

A

ATLL: peripheral blood (GSE3615)

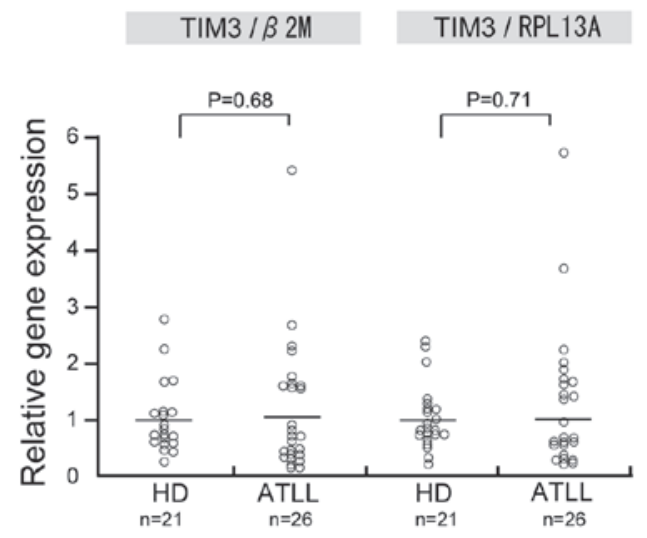

B PTCL: lymph node (GSE36172)

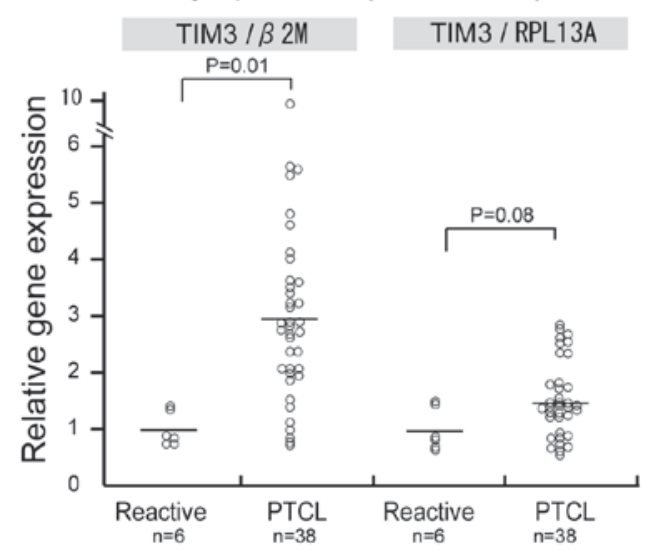

Figure 5. Database analysis of T cell Ig and mucin domain-containing molecule-3 (TIM-3) expression in human samples. Gene expression database of (A) peripheral blood cluster of differentiation 4-positive lymphocytes derived from healthy donors (HD) or patients with acute adult T-cell leukemia/lymphoma (ATLL) and of (B) lymph nodes diagnosed as reactive lymphadenitis and peripheral T-cell lymphoma (PTCL). $\beta-2$ microglobulin ( $\beta 2 \mathrm{M})$ and ribosomal protein L13a (RPL13A) were used as internal mRNA expression controls.

\section{Discussion}

In our previous study, a large number of TAMs were detected in cells from lymph node biopsy samples of almost all ATLL cases and a notably higher percentage of CD163-positive TAMs were found to be significantly linked to a worse clinical course (11). In vitro analysis showed that direct contact between ATLL cells and TAMs induced ATLL cell proliferation via several TAM-derived growth factors, including IL-6 and tumor necrosis factor- $\alpha$ (11). Based on these observations, we next considered that long-term co-culture with macrophages may change the characteristics of ATLL cells. In the present study, it was found that direct co-culture with macrophages induced chemoresistance in ATLL cells. This phenomenon was not observed when the ATLL cells were indirectly co-cultured with macrophages; therefore, direct contact with macrophages is suggested to play an important role in macrophage-induced chemoresistance in ATLL cells. This effect may be caused by several cytokines derived from macrophages that are strongly activated by direct contact with ATLL cells. The expression of intracellular adhesion molecule-1 (ICAM-1), membrane type M-CSF, P-selectin glycoprotein ligand-1 and unknown CD163 ligands on tumor cells is considered to be associated with macrophage activation in certain types of tumors $(13,14)$. Zheng et al demonstrated that 
an ICAM-1-blocking antibody reduced macrophage-induced chemoresistance in myeloma cells (15). Since ICAM-1 is also expressed in ATLL cells (16), ICAM-1 may be associated with macrophage-induced chemoresistance.

In the present study, the expression of TIM-3 in ATLL cells was induced by long-term co-culture with macrophages; however, the detailed molecular mechanisms by which this occurs remain unclear. Galectin-9, high mobility group protein B1 (HMGB-1) and phosphatidylserine are known to be ligands of TIM-3 (4-6), and our unpublished data that HMGB-1 is highly expressed in ATLL cells suggest that TIM-3 activation is induced by HMGB-1 that is derived from ATLL cells in an autocrine manner. Since no TIM-3 expression was observed in any of the lymphoma cell lines used in the present study in the absence of macrophage co-culture, unknown factors derived from activated macrophages or epigenetic change induced by direct contact with macrophages are considered to be necessary for the induction of TIM-3 in ATLL cells.

The present study also showed that TIM-3 expression is potentially associated with chemoresistance in in vivo and in vitro studies. However, a TIM-3 neutralizing antibody failed to affect the macrophage-induced chemoresistance in ATLL cells in an in vitro study (Horlad et al, unpublished data). This observation indicates the complexity of TIM-3 signaling. TIM-3 overexpression is also observed in activated lymphocytes and, as with cytotoxic T lymphocyte antigen 4 and programmed death 1, it is associated with tolerance and exhaustion (4-6). Recently, Huang et al demonstrated that carcinoembryonic antigen cell adhesion molecule 1 (CEACAM1) mediates TIM-3 activation and that blocking of the two molecules was required to induce lymphocyte activation (17). Thus, blocking of CEACAM1 and TIM-3 together may be necessary to impair the chemoresistance of TIM-3-positive ATLL cells.

By means of database analysis, in the present study, TIM-3 mRNA overexpression was observed in the samples of lymph node biopsies, but not in the peripheral CD4-positive lymphocytes of patients, as shown in Fig. 5. We suspect that direct contact between TAMs and lymphoma cells is observed only in lymph node samples of ATLL and that long-term direct contact with TAMs induced TIM-3 overexpression in the ATLL cells.

In conclusion, in an in vitro experiment, the present study found that direct contact with TAMs induced chemoresistance and TIM-3 expression in ATLL cells. It was also found that the TIM-3 expression found in the lymphoma cells of the ATLL biopsy samples was closely associated with the resistance to chemotherapy. In vitro study suggested that long-term direct contact with TAMs caused TIM-3 expression. Although TIM-3 overexpression induced the chemoresistance of ATLL cells, antibody-mediated neutralization of TIM-3 failed to improve the macrophage-induced ATLL chemoresistance. Although other unknown mechanisms are considered to be involved in macrophage-induced chemoresistance, TIM-3-related signals may be a useful target therapy for patients with ATLL. In addition, TIM-3 expression in biopsy samples of ATLL may be a good predictor of the effectiveness of chemotherapy.

\section{Acknowledgements}

The authors would like to thank Ms. Emi Kiyota, Mr. Osamu Nakamura, Ms. Yui Hayashida and Mr. Takenobu Nakagawa of the Department of Cell Pathology of Kumamoto University for their technical assistance. This study was supported by grants from the Ministry of Education, Culture, Sports, Science and Technology of Japan (no. 23790407 to Dr Yoshihiro Komohara, and no. 20390113 to Professor Motohiro Takeya).

\section{References}

1. Shirono K, Hattori T, Hata H, Nishimura $\mathrm{H}$ and Takatsuki K: Profiles of expression of activated cell antigens on peripheral blood and lymph node cells from different clinical stages of adult T-cell leukemia. Blood 73: 1664-1671, 1989.

2. Ohshima K: Pathological features of diseases associated with human T-cell leukemia virus type I. Cancer Sci 98: 772-778, 2007.

3. Shimoyama M: Diagnostic criteria and classification of clinical subtypes of adult T-cell leukaemia-lymphoma. A report from the Lymphoma Study Group (1984-87). Br J Haematol 79: 428-437, 1991.

4. Monney L, Sabatos CA, Gaglia JL, Ryu A, Waldner H, Chernova T, Manning S, Greenfield EA, Coyle AJ, Sobel RA, et al: Th1-specific cell surface protein Tim-3 regulates macrophage activation and severity of an autoimmune disease. Nature 415: 536-541, 2002.

5. Chiba S, Baghdadi M, Akiba H, Yoshiyama H, Kinoshita I, Dosaka-Akita H, Fujioka Y, Ohba Y, Gorman JV, Colgan JD, et al: Tumor-infiltrating DCs suppress nucleic acid-mediated innate immune responses through interactions between the receptor TIM-3 and the alarmin HMGB1. Nat Immunol 13: 832-842, 2012.

6. Tang D and Lotze MT: Tumor immunity times out: TIM-3 and HMGB1. Nat Immunol 13: 808-810, 2012.

7. Wiener Z, Kohalmi B, Pocza P, Jeager J, Tolgyesi G, Toth S, Gorbe E, Papp Z and Falus A: TIM-3 is expressed in melanoma cells and is upregulated in TGF-beta stimulated mast cells. J Invest Dermatol 127: 906-914, 2007.

8. Li H, Wu K, Tao K, Chen L, Zheng Q, Lu X, Liu J, Shi L, Liu C, Wang G and Zou W: Tim-3/galectin-9 signaling pathway mediates T-cell dysfunction and predicts poor prognosis in patients with hepatitis B virus-associated hepatocellular carcinoma. Hepatology 56: 1342-1351, 2012.

9. Zhuang X, Zhang X, Xia X, Zhang C, Liang X, Gao L, Zhang X and Ma C: Ectopic expression of TIM-3 in lung cancers: A potential independent prognostic factor for patients with NSCLC. Am J Clin Pathol 137: 978-985, 2012.

10. Komohara Y, Morita T, Annan DA, Horlad H, Ohnishi K, Yamada S, Nakayama T, Kitada S, Suzu S, Kinoshita I, et al: The coordinated actions of TIM-3 on cancer and myeloid cells in the regulation of tumorigenicity and clinical prognosis in clear cell renal cell carcinomas. Cancer Immunol Res 3: 999-1007, 2015.

11. Komohara Y, Niino D, Saito Y, Ohnishi K, Horlad H, Ohshima K and Takeya M: Clinical significance of CD163* tumor-associated macrophages in patients with adult T-cell leukemia/lymphoma. Cancer Sci 104: 945-951, 2013.

12. Takeda S, Maeda M, Morikawa S, Taniguchi Y, Yasunaga J, Nosaka K, Tanaka Y and Matsuoka M: Genetic and epigenetic inactivation of tax gene in adult T-cell leukemia cells. Int J Cancer 109: 559-567, 2004.

13. Komohara Y, Jinushi M and Takeya M: Clinical significance of macrophage heterogeneity in human malignant tumors. Cancer Sci 105: 1-8, 2014.

14. Komohara Y, Niino D, Ohnishi K, Ohshima K and Takeya M: Role of tumor-associated macrophages in hematological malignancies. Pathol Int 65: 170-176, 2015.

15. Zheng Y, Yang J, Qian J, Qiu P, Hanabuchi S, Lu Y, Wang Z, Liu Z, Li H, He J, et al: PSGL-1/selectin and ICAM-1/CD18 interactions are involved in macrophage-induced drug resistance in myeloma. Leukemia 27: 702-710, 2013.

16. Tanaka Y, Fukudome K, Hayashi M, Takagi S and Yoshie O: Induction of ICAM-1 and LFA-3 by Tax1 of human T-cell leukemia virus type 1 and mechanism of down-regulation of ICAM-1 or LFA-1 in adult-T-cell-leukemia cell lines. Int J Cancer 60: 554-561, 1995.

17. Huang YH, Zhu C, Kondo Y, Anderson AC, Gandhi A, Russell A, Dougan SK, Petersen BS, Melum E, Pertel T, et al: CEACAM1 regulates TIM-3-mediated tolerance and exhaustion. Nature 517: 386-390, 2015. 